\title{
Dispositivo de Baixo Custo para Deteç̧ão de Patologias da Visão
}

\author{
Rayanne M. C. Silveira ${ }^{1}$, João D. S. Almeida ${ }^{1}$, Jorge A. M. Teixeira ${ }^{1}$, \\ Ivana M. O. Maia $^{2}$, Anselmo C. Paiva ${ }^{1}$, Geraldo Braz Júnior ${ }^{1}$ \\ ${ }^{1}$ Núcleo de Computação Aplicada - Universidade Federal do Maranhão (UFMA) \\ São Luís - MA - Brasil \\ ${ }^{2}$ Departamento Acadêmico de Desenho - Instituto Federal do Maranhão (IFMA) \\ São Luís - MA - Brasil \\ rayanne.silveira@aluno.ecp.ufma.br, jdallysonenca.ufma.br
}

\begin{abstract}
The identification of vision pathologies in the first years of life (0 to 3 years) contributes to a more effective treatment. However, obtaining the diagnosis of patients in this age from traditional methods as the Snellen table, which requires patient collaboration, is not viable. The use of Photoscreeners to diagnose this group of patients has been an option, however these devices are very expensives. In this context, a similar and low cost device is proposed that can incorporate different computational methods to detect vision pathologies and allow to diagnose patients of all ages. The prototype has similar features compared to those on the market and cost 20 times less than the others devices.
\end{abstract}

Resumo. A identificação de patologias da visão nos primeiros anos de vida contribui para um tratamento mais eficaz. Entretanto, obter o diagnóstico de pacientes na faixa etária de 0 a 3 anos a partir de métodos tradicionais como a tabela de Snellen, que necessita da colaboração do paciente, é inviável. O uso de Photoscreeners para diagnosticar esse grupo de pacientes tem sido uma opção, entretanto o custo desses dispositivos é elevado. Neste contexto, propõese um dispositivo semelhante e de baixo custo que possa incorporar diferentes métodos computacionais para detectar patologias da visão e permita examinar pacientes de todas as idades. O protótipo apresenta funcionalidades semelhantes as dos dispositivos no mercado e apresenta um custo 20 vezes menor que o preço médio dos dispositivos existentes.

\section{Introdução}

Problemas da visão como o estrabismo, miopia, ambliopia e outros defeitos refrativos são cada vez mais comuns durante a infância. Segundo Harley e Nelson (2005), citados pelo autores Almeida et. al (2015), o estrabismo é uma patologia que afeta cerca de $4 \%$ da população. Especialistas acreditam que dos 3 aos 4 anos de idade, o sistema visual já esteja totalmente desenvolvido, mas ainda pode ser moldado até os 10 anos de idade. Com isso, para maior efetividade do tratamento o diagnóstico na fase de desenvolvimento é essencial para que o tratamento dessas patologias seja mais eficaz [Graziano and Leone 2005].

Uma das alternativas populares para detectar erros de refração e desalinhamento ocular (estrabismo) de forma simples e precisa ainda na infância, período cujo sistema 
visual ainda está em desenvolvimento, são os dispositivos de rastreamento de visão conhecidos como Photoscreeners ${ }^{1}$ [Clausen and Arnold 2007]. Diversos estudos como os de Forcina et. al (2017), Matta et. al (2009) e Singman et. al (2013) utilizaram diferentes modelos de Photoscreeners para avaliar a acurácia, sensibilidade e outros aspectos relevantes do desempenho dos mesmos, onde vários autores apontam esses dispositivos como uma ferramenta promissora.

Nesse sentido, seria oportuno que os programas de saúde pública priorizassem a identificação de alterações oftalmológicas nas crianças em idade pré-escolar e escolar, pois os principais problemas visuais, como estrabismo, glaucoma etc., podem diminuir a capacidade visual levando até à cegueira. A investigação de problemas oculares em crianças por meio de oftalmologistas mostra-se muito dispendiosa. Uma solução viável é a utilização de dispositivos de triagem auxiliada, para posterior avaliação por oftalmologistas.

Apesar de promissores esses dispositivos possuem um alto custo, o que dificulta o acesso e propagação desse tipo de tecnologia. Com o intuito de facilitar o acesso a dispositivos computacionais na área da saúde, propõe-se um dispositivo, com funcionalidades semelhantes as existentes nos Photoscreeners do mercado, de baixo custo, capaz de detectar precocemente patologias da visão baseado no processamento e análise de imagens.

Diante do exposto, o dispositivo em desenvolvimento poderá ser utilizado por profissionais que atuem na atenção básica de saúde e/ou docentes dos ensino básico e médio, favorecendo a detecção e tratamento precoce, a redução da incidência de baixa de visão, melhoria do rendimento escolar e desoneração do SUS pela diminuição dos custos financeiros pela não necessidade de oftalmologista.

O produto está sendo desenvolvido em projeto que possui aprovação do comitê de ética em pesquisa do Hospital Universitário Federal do Maranhão, através do parecer $\mathrm{N}^{\mathrm{o}}$ 1.242.814 para realização de estudos utilizando imagens de seres humanos.

\section{Dispositivos de Rastreamento}

A etapa inicial para o desenvolvimento do dispositivo proposto consiste em realizar pesquisa de mercado a fim de selecionar os dispositivos mais populares e que foram objetos de estudos de análise de desempenho, como o realizado por Forcina et. al (2017). Em uma análise dos dispositivos selecionados foi possível identificar os principais componentes e funcionalidades de cada um e traçar elementos básicos que o dispositivo proposto deve possuir.

O primeiro dispositivo analisado foi o Spot Vision Screener, desenvolvido pela Welch Allyn, que realiza captura de imagens por meio de uma câmera infravermelho, processa a imagem e a classifica a partir de critérios de referência do sistema. O dispositivo é portátil e pequeno, o que para diversos estudos é considerada uma grande vantagem [Silbert and Matta 2014].

O segundo dispositivo analisado, o Plusoptix, apresenta o mesmo princípio de funcionamento, onde através de uma câmera infravermelho obtém imagens para processamento e classificação. Um aspecto positivo é sua interface amigável, que contribui para focar a região dos olhos da criança durante o exame [Matta et al. 2008].

\footnotetext{
${ }^{1}$ São dispositivos que através da análise de imagens são capazes de detectar patologias da visão
} 
Além dos dispositivos já apresentados, a empresa Adaptica fornece um dispositivo com a mesma funcionalidade, o 2 WIN. Esse dispositivo possui o design semelhante a uma câmera fotográfica, assim como os demais apresentados realiza o diagnóstico a partir de imagens obtidas por uma câmera infravermelho e técnicas de processamento de imagens e classificação [Adaptica 2017].

Após o levantamento foi possível estabelecer os requisitos básicos do dispositivo proposto, com base nos pontos em comum de todos os dispositivos analisados, que engloba as funcionalidades da interface (software) e todos os elementos de entrada e saída de informações que o dispositivo deve possuir (hardware). Dentre os dispositivos de entrada é possível apontar: câmera infravermelho, tela touchscreen e botões de controle. Já os dispositivos de saída consiste em leds infravermelhos, leds de alto-brilho, emissor sonoro e display para exibição de informações.

Já a interface deve permitir o cadastro de pacientes, envio de relatórios e auxiliar na realização do exame, além de apresentar uma seção voltada para o administrador do dispositivo, que permita a inserção de novos métodos de exames, além da comunicação com um banco de dados local que será utilizado para gerenciar os dados dos exames realizados pelos pacientes.

\section{Desenvolvimento do Protótipo}

Após definidos os requisitos básicos do dispositivo iniciou-se a fase de desenvolvimento. Em uma visão geral, o dispositivo terá seu funcionamento baseado em 3 rotinas ilustradas na Figura 1, onde a rotina (A) consiste na configuração e aquisição de imagens. $\mathrm{Na}$ rotina (B) as imagens obtidas serão utilizadas para a execução de todos os métodos computacionais selecionados. Por fim, a rotina (C) refere-se ao relatório final do exame, apontando ou não a necessidade de encaminhar o paciente ao especialista.

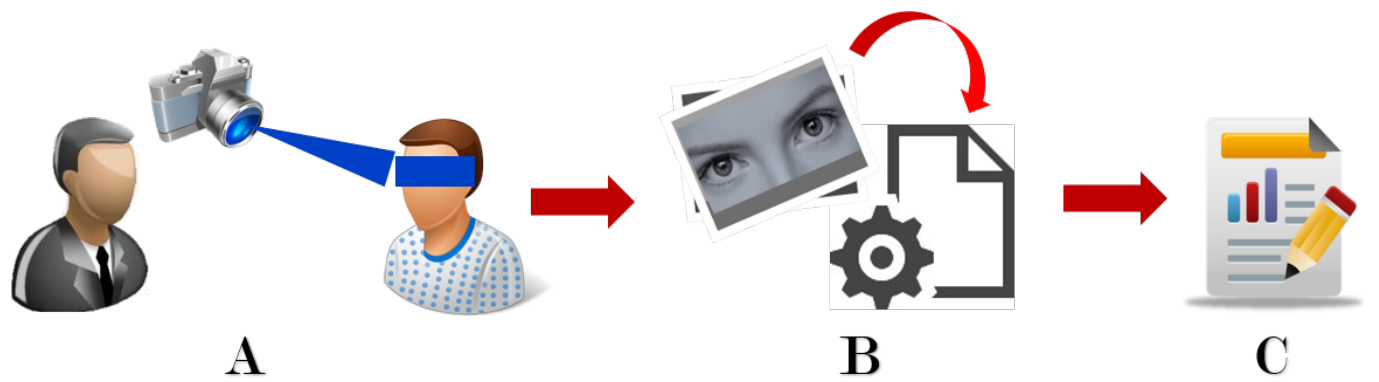

Figura 1. Sequência de rotinas do funcionamento do Dispositivo.

Logo, o desenvolvimento do protótipo foi dividido em duas etapas: desenvolvimento do software de gerenciamento e desenvolvimento do hardware. Iniciando-se pelo software, a interface proposta possui um banco de dados local, onde serão armazenados os dados dos pacientes, uma seção de gerenciamento de exames e a interface que será utilizada pelo operador durante os exames.

Além das operações de configuração e de execução do exame, o software proposto também é responsável pela emissão de um relatório contendo os resultados de cada um dos exames aplicados. É importante ressaltar que o dispositivo será aplicado para realizar 
triagem de pacientes, logo o resultado exibido no relatório pode ou não encaminhar o paciente para um especialista.

Dentre os gerenciadores de banco de dados disponíveis, neste projeto o SQLite foi utilizado, com base na sua simplicidade, velocidade e capacidade [Junyan et al. 2009]. O banco de dados desenvolvido é pequeno e possui finalidade apenas de armazenar informações dos pacientes e exames, relacionando-os com os pacientes correspondentes. $\mathrm{O}$ diagrama de entidade-relacionamento está ilustrado na Figura 2.

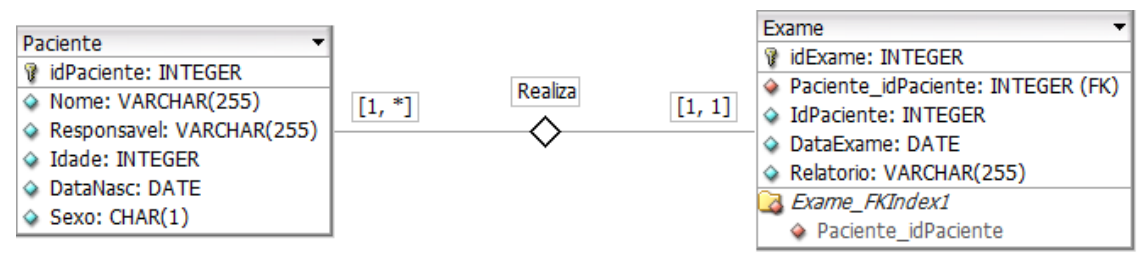

Figura 2. Diagrama Entidade-Relacionamento do Banco de dados.

Na Figura 3 está ilustrada a tela inicial do sistema, onde o operador pode optar por cadastrar o paciente, caso o mesmo não possua registro, pode iniciar o exame, caso o paciente já possua, ou então, pode gerenciar o sistema cadastrando ou removendo exames e pacientes da base de dados. Além dos requisitos funcionais, a interface foi desenvolvida para facilitar a operação do dispositivo, através de objetividade e clareza das funções disponíveis.

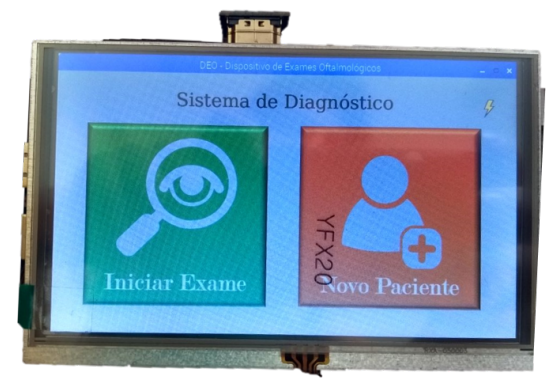

Figura 3. Interface do dispositivo.

Ainda em uma breve análise do software desenvolvido, é fundamental que o dispositivo tenha suporte a biblioteca OpenCV ${ }^{2}$, já que os métodos computacionais que serão incorporados ao sistema utilizam essa biblioteca.

Iniciando a análise do desenvolvimento do hardware, foram definidos os dispositivos que seriam utilizados para entrada e saída de informações, assim como o dispositivo que realizaria o processamento dessas informações. Com isso, definiu-se que seria utilizada a placa Raspberry como dispositivo de processamento, com isso periféricos como a câmera e display touchscreen foram escolhidos também.

Além dos periféricos já mencionados, alguns circuitos auxiliares tiveram que ser desenvolvidos, como o arranjo de infravermelhos, responsáveis por auxiliar a câmera utilizada. Para dar maior autonomia ao desenvolvedor do exame foram utilizados multiplexadores para controle de cada led e infravermelho individualmente.

\footnotetext{
${ }^{2}$ Biblioteca para o desenvolvimento de aplicações de Visão computacional.
} 


\section{Resultados e Discussões}

Alguns testes simulando a realização de um exame foram realizados no dispositivo, onde o funcionamento do sistema de gerenciamento foi verificado, assim como a inserção e busca de informações no banco de dados local. Além disso, a comunicação entre a interface e a câmera e os demais periféricos foram testados.

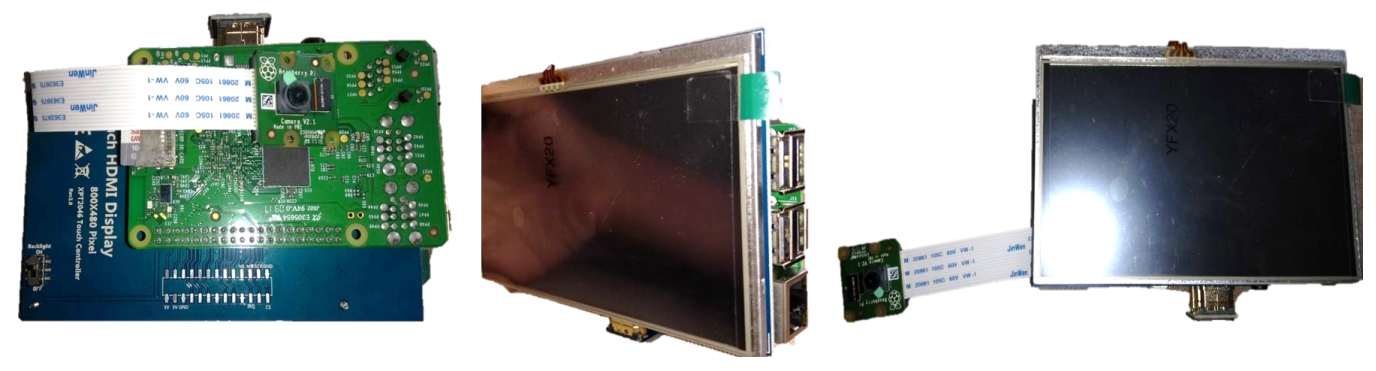

Figura 4. Protótipo Preliminar.

Outro fator a ser analisado é o custo parcial do hardware do protótipo, atualmente cerca de $\mathrm{R} \$ 1.020,00$, em uma comparação direta, o dispositivo possui um custo 20 vezes menor que o valor médio de mercado dos modelos mencionados neste trabalho. Na Figura 4 encontram-se algumas imagens do protótipo em fase de desenvolvimento, onde a estrutura básica, com a placa controladora (Raspberry pi 3), display touchscreen de 5 polegadas e câmera já estão integrados e interagindo corretamente com a interface gráfica implementada.

Quando finalizado, o dispositivo será avaliado através de aplicação de questionário de avaliação de usabilidade de sistemas (Post-Study System Usability Questionnaire - PSSUQ)[Redish 2017], para fins de validação da usabilidade do dispositivo tanto por parte do especialista quanto do paciente. Já os métodos computacionais incorporados serão validados individualmente através das métricas: acurácia, sensibilidade, especificidade e kappa [Wong and Lim 2011].

\section{Considerações Finais}

Com base na proposta central, o dispositivo apresenta baixo custo sem deixar de fornecer os mesmos recursos que os dispositivos do mercado. Entretanto, ainda há necessidade de testar a versão final do protótipo com métodos computacionais incorporados, para assim avaliar o desempenho total do dispositivo.

As próximas etapas consistem em desenvolver a carcaça do dispositivo, tornandoo visualmente atrativo e ergonomicamente de fácil manuseio. Após concluída esta etapa, deseja-se realizar testes do funcionamento do dispositivo em conjunto com métodos computacionais de detecção de patologia na visão.

A qualidade dos resultados obtidos a partir do desenvolvimento deste produto oferecerá subsídios para divulgar o dispositivo desenvolvido no mercado afim de facilitar a realização de triagens de pacientes realizadas por profissionais que atuem na atenção básica de saúde e docentes dos ensinos básicos e médios, favorecendo o diagnóstico e tratamento precoce. 


\section{Agradecimentos}

Os autores agradecem à FAPEMA (Processo: UNIVERSAL-01082/16) e ao CNPq (Processo: 423493/2016-7) pelo apoio financeiro.

\section{Referências}

Adaptica (2017). 2win binocular mobile refractometer and vision analyzer.

Almeida, J. D. S., Silva, A. C., Teixeira, J. A. M., Paiva, A. C., and Gattass, M. (2015). Computer-aided methodology for syndromic strabismus diagnosis. Journal of digital imaging, 28(4):462-473.

Clausen, M. M. and Arnold, R. W. (2007). Pediatric eye/vision screening. referral criteria for the pedia vision plus optix s 04 photoscreener compared to visual acuity and digital photoscreening. kindergarten computer photoscreening. Binocul Vis Strabismus $Q$, 22(2):83-9.

Forcina, B. D., Peterseim, M. M., Wilson, M. E., Cheeseman, E. W., Feldman, S., Marzolf, A. L., Wolf, B. J., and Trivedi, R. H. (2017). Performance of the spot vision screener in children younger than 3 years of age. American Journal of Ophthalmology, 178:79-83.

Graziano, R. M. and Leone, C. R. (2005). Problemas oftalmológicos mais frequentes e desenvolvimento visual do pré termo extremo. Jornal de pediatria, 81(1):95-100.

Harley, R. D., Nelson, L. B., and Olitsky, S. E. (2005). Harley's pediatric ophthalmology. Lippincott Williams \& Wilkins.

Junyan, L., Shiguo, X., and Yijie, L. (2009). Application research of embedded database sqlite. In Information Technology and Applications, 2009. IFITA'09. International Forum on, volume 2, pages 539-543. IEEE.

Matta, N. S., Arnold, R. W., Singman, E. L., and Silbert, D. I. (2009). Comparison between the plusoptix and mti photoscreeners. Archives of Ophthalmology, 127(12):1591-1595.

Matta, N. S., Singman, E. L., and Silbert, D. I. (2008). Performance of the plusoptix vision screener for the detection of amblyopia risk factors in children. Journal of American Association for Pediatric Ophthalmology and Strabismus, 12(5):490-492.

Redish, J. (2017). Quantifying the user experience: Practical statistics for user research.

Silbert, D. I. and Matta, N. S. (2014). Performance of the spot vision screener for the detection of amblyopia risk factors in children. Journal of American Association for Pediatric Ophthalmology and Strabismus, 18(2):169-172.

Singman, E., Matta, N., Tian, J., and Silbert, D. (2013). A comparison of referral criteria used by the plusoptix photoscreener. Strabismus, 21(3):190-194.

Wong, H. B. and Lim, G. H. (2011). Measures of diagnostic accuracy: sensitivity, specificity, ppv and npv. Proceedings of Singapore healthcare, 20(4):316-318. 